\title{
Gestión educativa: Tendencias de las políticas públicas educativas implementadas en Colombia
}

\author{
Andrea Aguilar- Barreto ${ }^{\text {a*}}$, Ysehidi Rocio Velandia -Riaño ${ }^{\mathrm{b}}$, Clara Paola \\ Aguilar- Barreto $^{c}$, Gerson Adriano Rincón- Álvarez ${ }^{\mathrm{d}}$ \\ aDoctora en Educación, Universidad Simón Bolívar, Cúcuta.(ID) , ${ }^{\mathrm{b}}$ Abogada en formación, \\ Universidad Simón Bolívar (DD'Magíster en Derecho Público, Universidad Simón Bolívar. (DD \\ dMagíster en Neuropsicología, Universidad Francisco de Paula Santander. (ID) \\ Forma de citar: Aguilar, A., Velandia, Y., Barreto, C. \& Álvarez, G. (2018). Gestión educativa: \\ Tendencias de las políticas públicas educativas implementadas en Colombia. Perspectivas, 2(2). 84-94.
}

Recibido: marzo 10 de 2017

Aceptado: junio 03 de 2017

\section{Palabras clave \\ Políticas públicas, políticas educativas públicas, calidad educativa}

* Autor para correspondencia a.aguilar@unisimonbolivar.edu.co
Resumen: Este artículo muestra los resultados de una investigación que se propuso identificar las principales tendencias en materia educativa que se han implementado en Colombia en el transcurso del siglo XX. Para ello se contextualiza la evolución histórica de las políticas públicas educativas colombianas durante el período e identificar las reformas educativas de mayor trascendencia en el país a fin de situar en un contexto más general las políticas públicas educativas actualmente vigentes. El trabajo se desarrolló mediante una revisión documental. La metodología utilizada fue cualitativa por cuanto estudia la realidad tal y como sucede y se procura otorgar sentido e interpretar los fenómenos de acuerdo con los significados que tienen para las personas implicadas. Del estudio se concluye que las políticas públicas del estado colombiano en torno a la educación han respondido a los propósitos de integración nacional, de valoración de la formación técnica como medio para potenciar la capacidad de la fuerza de trabajo, así como de homogenización de valores ideológicos básicos. 


\section{Keywords}

Public policies, public education policies, educational qualityforms of evaluation, metaevaluation

\section{Palavras chave}

Políticas públicas, políticas de educação pública, qualidade educacional

\section{Education Management: Trends in public education policies implemented in Colombia}

\begin{abstract}
This article shows the results of a research that aimed to identify the main trends in education that have been implemented in Colombia during the course of the 20th century. For this purpose, the historical evolution of Colombian public education policies during the period is contextualized and the most important educational reforms in the country are identified in order to place the current public education policies in a more general context. The work was developed through a documentary review. The methodology used was qualitative as it studies reality as it happens and seeks to give meaning and interpret the phenomena according to the meanings they have for the people involved. The study concludes that the public policies of the Colombian state regarding education have responded to the purposes of national integration, of valuing technical training as a way to enhance the capacity of the workforce, as well as the homogenization of ideological values basic.
\end{abstract}

\section{Gestão educacional: Tendências nas políticas de educação pública implementadas na Colômbia}

Resumo: Este artigo mostra os resultados de uma pesquisa que visou identificar as principais tendências em educação que foram implementadas na Colômbia no decurso do século XX. Para tanto, a evolução histórica das políticas coloniais de educação pública durante o período é contextualizada e as mais importantes reformas educacionais no país são identificadas para colocar as políticas atuais de educação pública em um contexto mais geral. O trabalho foi desenvolvido através de uma revisão documental. A metodologia utilizada foi qualitativa na medida em que estuda a realidade como acontece e procura dar sentido e interpretar os fenômenos de acordo com os significados que eles têm para as pessoas envolvidas. O estudo conclui que as políticas públicas do estado colombiano em matéria de educação responderam aos propósitos da integração nacional, a avaliação do treinamento técnico como meio de aumentar a capacidade da força de trabalho, bem como a homogeneização dos valores ideológicos básicos. 


\section{Introducción}

El propósito de este trabajo ha consistido en analizar cuáles han sido las principales tendencias en materia educativa en el siglo XX en Colombia. En este sentido, en primer lugar es preciso señalar que gran parte de las naciones latinoamericanas transitaron por procesos de modernización del sistema educativo durante la primera mitad del siglo XX, período en el cual intentaron copiar y aplicar los ideales del modelo de educación universal desarrollados en los países europeos a lo largo del siglo XIX. La velocidad con la que se produjeron importantes cambios políticos $\mathrm{y}$ sociales en los países latinoamericanos en el ese siglo configuró nuevos perfiles en el campo de la educación, cifrándose la esperanza en este medio como la posibilidad de inculcar valores ciudadanos acordes con la construcción de los nuevos estados modernos. En Colombia, este proceso tuvo un hito importante en el período de 1930-1946, cuando bajo los gobiernos sucesivos del partido liberal, conocidos como La República Liberal, la educación ocupó un importante lugar de orden nacional en torno al cual se concentró la atención de importantes sectores de opinión.

Colombia ha conocido una constante evolución industrial desde los primeros años del siglo XX, pero el proceso de modernización de las instituciones no se ha cumplido al mismo ritmo. Las exportaciones de café, flores, el litigio por el canal de Panamá, los préstamos y las inversiones extranjeras en petróleo, minería y servicios públicos, así como el florecimiento de industrias manufactureras y la inversión estatal en obras de infraestructura, son algunos de los elementos que constituyeron la base de la dinámica de estas transformaciones. Asimismo, la urbanización y la expansión del estilo de vida de las grandes ciudades al medio rural crearon expectativas en materia de participación social, salud, educación y servicios públicos.

Del estudio de estos procesos surge el interés por conocer cuáles han sido las tendencias que han influido en el diseño de las políticas públicas educativas en el desarrollo del siglo XX en Colombia, que es el motivo de la presente investigación.

El objetivo principal de esta investigación fue analizar la tendencia de las políticas públicas educativas implementadas en Colombia en el siglo $\mathrm{XX}$, para lo cual ha sido preciso contextualizar la evolución histórica de las políticas públicas educativas, identificar las reformas educativas de mayor trascendencia en este periodo y describir las políticas públicas educativas actualmente vigentes en el país.

\section{Materiales y métodos}

En el transcurso de la investigación seanalizaron las tendencias de las políticas públicas educativas implementadas en el siglo XX en Colombia por parte de las autoridades gubernamentales. Para ello se realizó una revisión documental sobre antecedentes y autores que han estudiado y tratado el tema a profundidad.

La investigación cualitativa estudia la realidad tal y como sucede, intentando otorgar e interpretar los fenómenos de acuerdo con los significados que tienen para las personas implicadas. Plantea, por un lado, que observadores competentes y cualificados pueden informar con objetividad, claridad $\mathrm{y}$ precisión acerca de sus propias observaciones del mundo social, así como de las experiencias de los demás. Por otro, los investigadores se aproximan a un sujeto real, un individuo real, que está presente en el mundo y que ofrece información sobre sus propias experiencias, opiniones y valores. Por medio de un conjunto de técnicas o métodos como las entrevistas, las historias de vida, el estudio de caso o el análisis documental, el investigador puede fundir sus observaciones con las observaciones aportadas por los otros. (Hernández Sampieri, et al. 1991)

El enfoque de investigación de este estudio fue interpretativo, pues según Gonzales Monteagudo (2000) la investigación que se fundamenta en este paradigma tendrá siempre como objetivo principal 
la comprensión de un fenómeno. En cuanto al tipo de investigación, fue documental (Hurtado de Barrera, 2000). A esta revisión siguió una contextualización de la evolución de las políticas públicas educativas, destacando entre ellas las reformas educativas de mayor trascendencia en Colombia, para finalmente estudiar las políticas públicas educativas vigentes en el país.

\section{Fundamentación teórica}

\subsection{El sistema educativo colombiano}

En Colombia, la educación en Colombia se concibe como un derecho ciudadano y una prioridad del gobierno. De conformidad con la Constitución de 1991 y la Ley General de Educación de 1994, todos los colombianos tienen derecho a acceder a la educación para su desarrollo personal y para el beneficio de la sociedad. La educación obligatoria actualmente es de 10 años, desde los 5 hasta los 15 años de edad. El sistema educativo, por otra parte, se encuentra organizado en cuatro etapas importantes. La Educación Inicial y Atención Integral a la Primera Infancia (EIAIPI) incluye servicios para los niños desde el nacimiento hasta los 6 años. En teoría, los estudiantes entran al sistema educativo en el año de transición (Grado 0, o a los 5 años de edad). La educación básica comprende nueve años (Grados 1 a 9, para niños de 6 a 14 años) e incluye cinco años de educación primaria y cuatro años de básica secundaria. La educación media dura dos años (Grados 10 y 11, para jóvenes de 15 y 16 años), un año menos que el promedio de la OCDE de tres años. El sistema de educación superior del país es especialmente complejo, con una gran variedad de proveedores y múltiples programas de distintas duraciones y niveles. Los estudiantes de Colombia son menores que sus pares de la OCDE cuando ingresan a la educación superior y es probable que hayan recibido menos años de educación (Ministerio de Educacion Nacional, 2016).
Actualmente, la educación y formación para niños entre 3 y 5 años está regulada dentro del plan nacional de educación. En lo que respecta a la educación básica, actualmente hay matriculados 7,5 millones de estudiantes en aproximadamente 50.000 sedes que existen en todo el territorio nacional. Es importante resaltar que la mayoría de estas sedes tiene un horario de clases entre 5 y 6 horas diarias, por lo que el gobierno nacional viene realizando una serie de esfuerzos para poner en funcionamiento una jornada escolar única durante la cual se incluya el suministro de alimentación a los jóvenes pertenecientes a las instituciones oficiales del país.

En lo que respecta a la educación superior, en Colombia hay más de 288 instituciones dedicadas a ofertarestudios superiores.E128\%sonuniversidades, el $42 \%$ son instituciones universitarias y el $18 \%$ son instituciones tecnológicas; el 15\% restante está formado por instituciones técnicas que permiten a los jóvenes dar continuidad a sus estudios de formación superior.

\subsection{Nuevas políticas públicas educativas en Colombia}

Una serie de medidas, desde la prestación de educación pública gratuita hasta grandes inversiones en infraestructura y recursos educativos, han permitido incrementar en un tiempo relativamente corto la cobertura de educación secundaria, lo cual ha permitido disminuir la deserción escolar y aumentar el número de estudiantes en los diferentes sectores del país. Actualmente uno de cada diez estudiantes que terminan la educación primaria no siguen estudiando la secundaria, situación que limita el mejoramiento de la calidad de vida de estos adolescentes y en el futuro de sus familias (Ministerio de Educacion Nacional, 2016).

\subsection{Evolución y desarrollo de la gestión educativa}

El siglo XX inició con la guerra de los mil días, que transcurrió entre 1899 y 1902. Como 
consecuencia, no se pudo iniciar deforma más caótica en materia educativa. Entre los resultados de esta guerra estuvo que muchos colegios y escuelas fueran destruidas y numerosos estudiantes no pudieron volver a sus clases. El país retrocedió en materia educativa, lo cual se evidenció sobre todo en los altos índices de deserción escolar y analfabetismo. En materia económica, la deuda externa se incrementó (Ramírez y Tellez, 2006), con lo cual la inversión pública, en general, pero sobre todo en lo que corresponde a la educación, decayó.

Para comprender mejor este inicio de siglo es necesario retroceder un par de décadas y contextualizar la situación. La Constitución de 1886 expresaba en su artículo 41 que "La educación pública será organizada y dirigida en concordancia con la Religión Católica." Como resulta evidente, la influencia de la iglesia católica en la vida social de la época era importantísima. Esta facultad de influencia otorgada a la Iglesia por la Constitución se ratifica con el concordato celebrado el 31 de diciembre de 1887 (González, 1939) entre la Santa Sede y la República de Colombia mediante Plenipotenciario designado por el papa León XIII y el Plenipotenciario nombrado por Rafael Núñez, presidente de Colombia de la época, dentro del cual se mencionaba entre otras cosas, lo siguiente:

Artículo 12.

En las universidades y en los colegios, en las escuelas y en los demás centros de enseñanza, la educación e instrucción pública se organizará y dirigirá en conformidad con los dogmas y la moral de la Religión Católica. La enseñanza religiosa será obligatoria en tales centros, y se observarán en ellos las prácticas piadosas de la Religión Católica.

\section{Artículo 13.}

Por consiguiente, en dichos centros de enseñanza los respectivos Ordinarios diocesanos, ya por sí, ya por medio de delegados especiales, ejercerán el derecho en lo que se refiere a la religión y la moral, de inspección y de revisión de textos. El arzobispo de Bogotá designará los libros que han de servir de texto para religión y la moral en las universidades; y con el fin de asegurar la uniformidad de la enseñanza en las materias indicadas, este Prelado de acuerdo con los otros Ordinarios diocesanos, elegirá los textos para los demás planteles de enseñanza oficial. El Gobierno impedirá que, en el desempeño de asignaturas literarias, científicas $\mathrm{y}$, en general, en todos los ramos de instrucción, se propaguen ideas contrarias al dogma católico y al respeto y veneración debidos a la Iglesia.

\section{Artículo 14}

En el caso de que la enseñanza de la religión y la moral, a pesar de las órdenes y prevenciones del Gobierno, no sea conforme a la doctrina católica, el respectivo Ordinario diocesano podrá retirar a los Profesores o Maestros la facultad de enseñar tales materias.

Para 1903, se inicia el mandato de Marroquín, quien con la Ley 39 (Congreso de la Republica, 1903) estableció las bases del sistema educativo del país para los primeros treinta años del siglo XX (Ramírez y Tellez, 2006). Con el fin de mejorar el sistema educativo establece la división de las etapas de la educación en primaria, secundaria, profesional, artística e industrial.

Entre 1910 y 1925 se desarrollan una serie de acontecimientos que influirían en el desarrollo industrial del país (periodo de gobierno del Pedro Nel Ospina). En cuanto a la educación, resaltan en esta época los siguientes hechos: En 1917 se celebra el $1^{\circ}$ Congreso Pedagógico Nacional. Se inicia también la escuela nueva de Agustín Nieto Caballero, quien trajo de visita al pedagogo Ovidio Decroly, creador de la escuela activa, cuyo método consistía en sentir, pensar y expresar. "La escuela ha de ser del niño, no el niño para la escuela." También arribó en esta época la misión alemana, que tuvo como fin orientar la posible Reforma Educativa que, sin embargo, resultó fallida. Finalizando este periodo se crea una de las leyes más importantes del siglo, la Ley 56 de 1927 (Congreso de la Republica, 
1927), por la cual, entre otras cosas, se establece que el Ministerio de Instrucción y Salubridad Pública pase a ser llamado Ministerio de Educación Nacional. Esta ley también obligó a los padres a ofrecer un mínimo de educación a sus hijos.

En la década de los años 20, El Ministerio de Educación Nacional fue el organismo a través del cual el Estado buscó, al igual que en otras órbitas de lo social, tener mayor presencia. Aunque su influencia distaría de las pretensiones de control y centralización que tenían los gobernantes, las diversas fuerzas comprometidas en la educación tuvieron que tenerlo en cuenta y someterse en muchos de los casos a sus disposiciones. Por tal motivo se reglamentó la Ley 56 de 1927 (Congreso de la Republica, 1927) que reguló la reorganización del Ministerio y su cambio de nombre, pasando de ser Ministerio de Instrucción y Salud Pública para convertirse en Ministerio de Educación Nacional, con la cual se pretendía dar mayor efectividad a las decisiones tomadas por esta entidad.

En este periodo, Colombia inicia su gran transformación hacia la actividad económica, la cual estuvo siempre ligada al desarrollo educativo. El aumento de la población en todo el territorio nacional obligó al Estado a fortalecer su sistema educativo, concediéndole un lugar más relevante.

Sin embargo, no se fue una época fácil, ya que por influencia de los partidos políticos se inculcaba a la sociedad una serie de pensamientos mercantilistas vinculados en última instancia con intereses privados, lo que dificultaba al Ministerio imponer su punto de vista para lograr unidad de criterios y obtener en algunos aspectos consenso ideológico. Diversos fueron los mecanismos utilizados con este propósito: se legisló de manera explícita sobre la educación secundaria y se condicionó la expedición de títulos a la aprobación oficial, se tuvo un control directo sobre las instituciones formadoras de docentes, la campaña de cultura aldeana llevó al sector rural los planteamientos del Ministerio y se editaron algunas publicaciones en las que se impartió un punto de vista sobre los fenómenos culturales y educativos (Herrera, 1984).
Posteriormente, el Ministerio de Educación Nacional, mediante la expresión cultural, dio a conocer su visión sobre educación y cultura, creando bibliotecas y estimulando la generación de hábitos de lectura, a la vez que se promovieron conferencias culturales, espectáculos públicos, así como la adquisición de cinematógrafos y aparatos radiofónicos.

De esta fecha datan algunas publicaciones de periódicos y de libros memorables. Tal es el caso de la Revista del Maestro, la Revista Rin Rin, La Revista de las Indias y otras muchas publicaciones. Para ese momento la tendencia que destacó fue la de dar publicidad y propagar una cultura de hábitos de lectura sana entre toda la población, niños, jóvenes y adultos. De la misma forma, se fomentó la construcción y uso de las bibliotecas, las cuales fueron dotadas con obras editadas por el Ministerio. Estas publicaciones cubrieron cuatro series: la primera de cartillas y manuales técnicos sobre nociones básicas de agricultura, alimentación, higiene, carpintería, etc.; la segunda, conformada por una colección de literatura universal en la que se incluyeron obras como el Quijote, la Divina Comedia, la Odisea, la Ilíada, la Eneida; la tercera serie era de literatura colombiana y estaba constituida por un total de cien obras; la última serie no poseía un criterio unificador tan claro como las anteriores y estuvo compuesta por obras aisladas de distintas materias.

La década del 20 fue una época difícil. En ella se consolidaron algunos acuerdos ya existentes entre la iglesia católica y el gobierno, la educación primaria y secundaria era lo más destacado, y se carecía de estudios técnicos y comerciales en la educación media técnica. El avance en materia educativa fue muy lento durante este lapso (Helg, 1987).

Los planteamientos en torno a la formación educativa de los sectores más populares tuvieron gran importancia en las décadas siguientes, durante las cuales existió la preocupación por ampliar la oferta educativa a una franja mayor de la población. Para esta época se logró hacer un acuerdo sobre el tema educativo entre los diferentes sectores de la 
sociedad. Se trataba de un objetivo razonable para un momento de evoluciones que ocurrían en el país como consecuencia de la modernización de sus estructuras e industrias, momento que señalaba la urgencia de elaborar e inculcar valores ideológicos que legitimaran la nueva situación económica, política y social. De esta forma se abrió paso el concepto de educación para los sectores más populares con el que se pretendió formar hombres útiles a la sociedad, con una moral y una conducta adecuadas a la categoría de ciudadanos, aptos para producir económicamente y colaborar con el interés general de la nación.

El presidente de ese entonces, López Pumarejo, tomó la decisión de hacer y de ver a la educación del pueblo como el núcleo central de la política gubernamental. Para lograr este objetivo se desarrollaron acciones que se contó con recursos más amplios que el aula escolar, de modo que los sectores de la población que no asistían a la escuela también pudieran recibir algún tipo de educación. Una política pública destacada en esta época fue la campaña de cultura aldeana: se crearon las bibliotecas populares y se contrataron maestros ambulantes que viajaban por todo el territorio nacional; dichas iniciativas recogieron en parte las experiencias mexicana y española sobre educación popular que se ponían en práctica en aquellos países por este mismo período (Herrera, 1984).

El periodo trascurrido entre 1946 y 1957 llevó al país a una de las formas más agresivas y revolucionarias de lucha de clases, incluso dentro del mismo partido político liberal, que se dividía entre sí, en liberalismo político y liberalismo económico. Las facciones estaban liderizadas respectivamente por los dirigentes de turno Ospina Pérez y Gaitán. En materia de educación las cosas se mantenían esencialmente iguales: persistían problemas antiguos tales como la falta de aulas, el bajo nivel de calidad de vida, la imposición de trabajo a temprana edad, la dispersión geográfica, deficientes condiciones de salud o la carencia o deficiente preparación de maestros. Se mantenían, sin embargo, esperanzas de cambio, y muchos educadores trabaron arduamente por conseguirlos. Aunque la lucha de algunos era incansable y tenaz la creación de una normatividad que permitiera reorganizar el sistema educativo y así poder desarrollar un plan integral de organismos de planeación, organización, dirección y control educativo, para el momento parecía casi imposible de alcanzar, pues cuando se contaba con el presupuesto suficiente no se contaba con el apoyo y la aprobación del gobierno de turno que concediera fuerza vinculante a cada uno de estos proyectos; o sino, ocurría lo contrario, de modo que los avances seguían quedando plasmados solo en papel.

En 1962 se creó la Federación Colombiana de Educadores sobre la base de Sindicatos Departamentales, bajo la premisa de procurar estabilidad y mejora para los maestros, lo que de cierta manera fue un obstáculo para las negociaciones con la Nación. En 1968 se realizó una reforma educativa que impulsó una transformación administrativa en el sector educativo que permitiera mejorar el esquema imperante de descentralización de la administración y la centralización de la política educativa, que tenía como objetivo anular las falencias de la constitución del 86 en cuanto a las competencias y facultades a nivel Departamental, Municipal y Nacional en el campo educativo. Para este periodo se crea la Junta de Escalafón Departamentales y los Fondos Educativos Regionales.

En la última década del siglo $\mathrm{XX}$, con la entrada en vigencia de la carta política de 1991 y la Ley General de Educación, Ley 115 de 1994, se ha establecido la política educativa en función del ideal de ciudadano que esas mismas normas proyectan a través de los derechos y deberes que se les atribuyen. En esencia, toda la legislación y la política administrativa colombianas consagran el derecho fundamental de las personas a acceder a la educación y asumen la responsabilidad de garantizar la calidad del servicio educativo, lo mismo que su prestación a todos los sectores y grupos humanos. En la normatividad se perfila, igualmente, una política progresiva, que asegure a todos un desarrollo personal y colectivo en beneficio de toda la sociedad (Conferencia Internacional de Educación, 2001) 


\section{Las reformas educativas de mayor trascendencia en Colombia durante el siglo XX}

En estas décadas la educación tomó una gran relevancia, básicamente entre los sectores populares de la sociedad, es decir, en los estratos más bajos. En efecto, las instituciones del gobierno nacional se preocupan y ocupan en ampliar la cobertura escolar de modo que las políticas públicas educativas fueran más efectivas. Uno de los objetivos fundamentales de momento fue la erradicación del analfabetismo. La creación de un consenso social fue otro de los objetivos centrales de la educación popular, objetivo coherente con el momento de transformaciones que ocurrían en el país como consecuencia de la modernización de sus estructuras, momento que señalaba la urgencia de elaborar e inculcar valores ideológicos que legitimaran la nueva situación económica, política y social. Fue así como se acogió en la sociedad el concepto de crear hombres útiles para el mejoramiento de la sociedad, acordes con las necesidades de la sociedad y aptos para contribuir económicamente en el desarrollo social. En este periodo la educación popular se vio enfrentada a un gran problema relacionado con las condiciones socioeconómicas de la población. Se llegó a pensar en la necesidad de restaurar en primer término la condición fisiológica de la población escolar, entendida como la atención al régimen alimenticio, la nutrición, la salud física y mental, lo cual se consideró como premisa para la adquisición de los conocimientos escolares (Herrera, 1984).

Las órdenes dadas desde el nivel central se focalizaron en el sentido de dar prioridad a la creación de escuelas y construcción de edificaciones para el funcionamiento de aulas de clase $\mathrm{y}$, por supuesto, dotados de útiles escolares.

Los programas educativos diseñados por el Ministerio buscaron adecuarse a las teorías de la escuela activa europea y especialmente a los postulados de Decroly, quien visitó al país en 1925 y dictó numerosas charlas sobre su método de enseñanza globalizada y con base en los centros de interés. Decroly aseguraba que el niño en su proceso de conocimiento captaba el todo antes que las partes, motivo por el cual había que enseñarle sobre la base del conjunto, de lo global. Al mismo tiempo, se apoyaba en una pedagogía del interés que hallaba su punto de apoyo en las motivaciones propias del niño y situaba la utilidad de su formación individual al servicio del engranaje social.

En lo que respecta a la educación secundaria, en esta época, como en casi todas, las políticas públicas educativas estatales han sido insuficientes y en ocasiones inapropiadas. Para la época existían muy pocas instituciones oficiales, y las que había, estaban en su mayoría bajo la regencia de comunidades religiosas. Las instituciones privadas eran mayoría, por lo cual el Estado decidió apoyar al sector privado incluyendo una serie de criterios para procurar su normalización, Al mismo tiempo, se puso en marcha una serie de medidas que buscaban dar mayor seriedad y calidad a la educación impartida en la fecha por parte del Ministerio de Educación Nacional.

En materia de educación superior se produjo una reforma universitaria caracterizada por una mayor inversión presupuestaria y la búsqueda de mayor coherencia entre las disposiciones legislativas. En los diversos planteamientos se encontraba viva la preocupación por formar un cuerpo de intelectuales que elaborara, desde nuevas perspectivas, teorías explicativas sobre diversos ámbitos de la realidad nacional, y que se desempeñara en las ocupaciones surgidas como consecuencia de una sociedad en crecimiento, fenómenos que dieron paso a la diversificación de las profesiones académicas. Más que un cambio en las estructuras materiales, la reforma universitaria requería de una transformación en el orden ideológico, que le permitiera cumplir con los fines propios de la educación profesional, los cuales se consideraron de carácter científico, social y académico (Herrera, 1984).

La Universidad Nacional fue el centro de la reforma universitaria. Se consideró que ella debía dar orientación a las cuatro universidades oficiales que existían en el país, por lo que el gobierno dispuso que estas se sometieran al régimen dictado por aquella. Estas universidades estaban situadas 
en las ciudades de Medellín (Universidad de Antioquia, 1822), Pasto (Universidad de Nariño, 1904), Popayán (Universidad del Cauca, 1827), y Cartagena (Universidad de Cartagena, 1827). Para la época la tendencia política era netamente liberal.

Unaspectoquedesempeñó un papel fundamental en estos procesos fue la profesionalización del magisterio. Para el momento se dictaron una serie de pautas destinadas a fortalecer las instituciones formadoras de docentes y regular el ejercicio de la profesión. Igualmente se empezó a trabajar en la estructuración del magisterio como sector gremial. En general, estos elementos confluyeron en lo que podría denominarse la profesionalización del magisterio en Colombia.

Así como se avanzaba en las políticas públicas educativas desde la década de los años 20, también surgían problemas que se generaron en torno a la reforma educativa. Los intentos gubernamentales por ejercer control sobre órbitas en las que tradicionalmente había ejercido potestad la iglesia católica, generaron numerosos enfrentamientos con los sectores tradicionales de la sociedad. En ellos se pusieron en conocimiento las concepciones en torno a los principios ideológicos que deberían guiar las coordenadas morales y doctrinarias de la nación colombiana y junto con ella las de la educación. Sectores delcleroydelpartido conservadorcalificaron la contienda entre los poderes civil y eclesiástico, como había ocurrido en España y México, países en los que se marcó una separación tajante entre las dos esferas. Este no fue estrictamente el caso de Colombia, puesto que el Estado, más que presentar posiciones anticatólicas, pretendió regular algunas esferas de la sociedad. La iglesia católica se opuso al proyecto de reforma constitucional, presentado por el gobierno nacional en 1936, que pretendía consagrar en la carta constitucional, el cambio en la dirección de las funciones estatales y reflejar una sociedad moderna en el campo ideológico. Sin duda que estas modificaciones lograron una mayor autonomía estatal sobre las esferas política, social y económica.
En la década de los años 50, cuando la segunda guerra mundial había llegado a su fin, el crecimiento del país, gracias a su fortalecimiento institucional, permitió que el Estado patrocinara la educación media y técnica. Por su parte, la iglesia católica jugó un papel fundamental en la organización del sector educativo en el país. Finalmente, en este periodo la visita se brigadas de otros países, que arribaron para cumplir misiones específicas, contribuyeron enormemente al crecimiento educativo de Colombia (Helg, 1987).

Más adelante, en las décadas del 60 y 70, este crecimiento educativo se frenó parcialmente. Las políticas públicas educativas de los gobiernos de turno reflejaban el retraso educativo que presentaba el país a la fecha, de forma que se vio la necesidad de generar políticas activas para promover su desarrollo. Así, el presidente Alfonso López Michelsen dio prioridad a la globalización de la educación primaria tanto en las áreas urbanas como en las rurales, buscando implementar métodos y estrategias internacionales para llegar a la población menos favorecida. Asimismo, esta administración considera como una de las causas para la baja escolaridad la falta de capacidad física para recibir a todos los aspirantes y plantea, tanto en educación primaria como secundaria, la construcción y dotación de establecimientos y la provisión de nuevas plazas para docentes (Ramírez y Téllez, 2006).

\section{Las políticas públicas educativas vigentes en Colombia en el 2016}

En Colombia se encuentra actualmente vigente el Plan Nacional Decenal de Educación, que rige y regirá la educación en Colombia durante los 10 años que comprende. El alcance de la formulación del PNDE 2016-2026 consiste en contar con un plan innovador, incluyente, de construcción colectiva y regional, que plantee los fundamentos necesarios para que Colombia tenga más y mejores oportunidades en 2026. De tal manera, se generará un gran acuerdo nacional que comprometa al gobierno, los diferentes sectores de la sociedad y la 
ciudadanía, para avanzar en las transformaciones que Colombia requiere desde la educación (Ministerio de Educación Nacional, 2016).

$\mathrm{Su}$ construcción parte de una amplia participación municipal, departamental, regional y nacional, en la cual los colombianos, incluyendo los de la base de la pirámide, expertos reconocidos en materia de educación, niños, jóvenes, adultos, mujeres, hombres, quienes viven en las ciudades, en zonas rurales, en condición de discapacidad, afrodescendientes, raizales, palanqueros, indígenas, campesinos, Rom, inmigrantes, colombianos en el exterior e iglesias, lo construirán desde su diseño hasta su redacción final.

Como toda política pública, debe tener un sustento jurídico, y este recae sobre el artículo 72 de la Ley 115 de 1994, Ley General de Educación. El PNDE se puede definir como el conjunto de propósitos, objetivos y metas que expresan la voluntad del país en materia educativa. Así, el objetivo de la formulación es generar un gran acuerdo nacional que comprometa al gobierno, los diferentes sectores de la sociedad y la ciudadanía en general, para avanzar en las transformaciones que la educación colombiana necesita.

\section{Conclusiones}

En el desarrollo del presente trabajo investigativo se pudo analizar la tendencia de las políticas públicas educativas en Colombia durante el siglo XX. Para ello fue necesario realizar una contextualización de la evolución histórica y sus principales reformas, iniciando en la década de 1900. Allí, como consecuencia de la guerra de los mil días, el país atravesaba por una serie de crisis que centraban la atención del país en todo menos en el desarrollo o progreso educativo, pues para esta época era más importante superar la fuerte crisis económica.

En el periodo de la República Liberal se alcanzaron logros significativos. Para la década del cincuenta, por ejemplo, el sistema educativo, pese a sus grandes deficiencias, se hallaba bastante estructurado, al tiempo que el gobierno, a través de Ministerio de Educación buscaba alcanzar una mayor racionalización y centralización en la planeación, administración, ejecución y supervisión de los hechos educativos. No obstante, es preciso enfatizar el escaso radio de acción que alcanzó el Ministerio en estas décadas, debido a los intereses regionales y de partido, así como a los escasos recursos financieros que se le asignaron, lo cual condujo a que en muchas ocasiones los proyectos fueran más ambiciosos que sus aplicaciones reales.

El Estado fue avanzando en el sentido de las disposiciones sobre planes y programas de estudio, tanto sobre las finalidades de la educación como en lo que respecta a la difusión de nuevas teorías pedagógicas. Como consecuencia, se empezó a conformar un discurso pedagógico oficial que no existía en períodos anteriores, con independencia del religioso, en el que se deja entrever la voluntad de institucionalizar, legitimar y controlar las prácticas pedagógicas. Para ello fue de gran importancia la creación de la inspección escolar nacional. Lo anterior no significa, sin embargo, que el papel del Estado haya sido tan enérgico como para asumir las funciones que le competían en correspondencia con un estado moderno.

En términos generales se puede decir que los elementos culturales difundidos obedecieron a propósitos de integración nacional, de valoración de la formación técnica como medio para potenciar la capacidad de la fuerza de trabajo, así como de homogenización de valores ideológicos básicos entre las masas de migrantes urbanos. A la educación se le asignó un papel importante como modeladora de actitudes y comportamientos. Esta educación traspasó el ámbito escolar para tejer una red que abarcaba las costumbres y hábitos de la población e introducirse en la esfera de la socialización.

Sin lugar a dudas, la economía y la educación jugaron un papel fundamental en el periodo inicial del siglo XX, debido a que la educación potenció en algunas regiones del país la economía a fin de contribuir con el despegue financiero en sectores geográficamente distantes de la capital del país. 
Finalmente, la impronta de la iglesia católica en la dirección del sector educativo de Colombia ha sido una de las causas más destacadas de su tradicional conservadurismo.

\section{Referencias Bibliográficas}

Asamblea Nacional Constituyente. (05 de agosto de 1986). Constitucion Política de 1986. Cúcuta, Colombia.

Asamblea Nacional Constituyente. (04 de julio de 1991). Constitucion Política de 1991. Bogotá, Colombia.

Conferencia Internacional de Educacion. (2001). Informe nacional sobre el desarrollo de la educacion en colombia. Ginebra: UNESCO.

Congreso de la República. (30 de Octubre de 1903). Ley 39 de 1903. Diario oficial número 11, 931. Bogotá, Colombia.

Congreso de la República. (10 de Noviembre de 1927). Ley 56 de 1927. Por la cual se dictan algunas disposiciones sobre instruccion pública. Bogotá, Colombia.

Congreso de la República. (10 de Noviembre de 1927). Ley 56 de 1927. Por la cual se dictan algunas disposiciones sobre instruccion publica. Bogotá, Colombia.

Congreso de la República. (21 de Diciembre de 2001). Diario Oficial No 44.654 de 21 de diciembre de 2001. Por la cual se dictan normas orgánicas en materia de recursos y competencias de conformidad con los artículos 151, 288, 356 y 357 (Acto Legislativo 01 de 2001) de la Constitución Política y se dictan otras disposiciones. Bogotá, Colombia.

Congreso de la República. (15 de marzo de 2013). Por la cual se crea el sistema nacional de convivencia escolar y formación para el ejercicio de los derechos humanos, la educación para la sexualidad y la prevención ymitigación de la violencia escolar. Bogota, Colombia.

Gonzáles Monteagudo, J. (2000). El paradigma interpretativo en la investigacion social $y$ educativa: nuevas respuestas para viejos interrogantes. Cuestiones pedagógicas: Revista de ciencias de la educación, 15, 227-246.

Helg, A. (1987). El desarrollo de la educación primaria y secundaria. Bogotá: Pysen Colombia.

Hernández Sampieri, R. et al. (1991). Metodologia de la investigacion. México: Trillas.

Herrera, M. C. (1993). Historia de la educacion en Colombia. La república liberal y la modernizacion de la educacion: 1930-1946. Revista Colombiana de educación, 26. Disponible en file://C:/Users/ FFB/Downloads/5297-13881-1-PB\%20(1).pdf.

Hurtado de Barrera, J. (2000). Metodología de la investigacion holistica. Caracas: Fundacion SYPAL.

Ministerio de Educacion Nacional. (2016). Plan Nacional Decenal de Educación. Bogotá: Ministerio.

Ramírez, M. T., y Téllez, J. P. (2006). La educación primaria y secundaria en Colombia en el siglo XX . Bogotá: Banco de la República.

Vacca, E. S. (2014). La deserción escolar en el contexto rural colombiano. Caso Guateque Boyacá. Trabajo de grado no publicado para obtener el título de Maestría en Educación. Bogotá: Universidad Pedagógica Nacional. 